\title{
ULTRAMICROSCOPIC CHARACTERISTICS OF ERYTHROCYTES IN INDIVIDUALS WITH BORRELIOSIS, RHEUMATOID ARTHRITIS AND TOXOPLASMOSIS AS A MARKER, COMORBID OR CONCOMITANT PATHOLOGY FOR BABESIOSIS
}

DOI: 10.36740/WLek202109107

\author{
Inna I. Torianyk \\ STATE INSTITUTION «MECHNIKOV INSTITUTE OF MICROBIOLOGY AND IMMUNOLOGY OF THE NATIONAL ACADEMY OF MEDICAL SCIENCES OF \\ UKRAINE», KHARKIV, UKRAINE
}

\begin{abstract}
The aim is to determine the ultramicroscopic characteristics of erythrocytes in individuals with rheumatoid arthritis, borreliosis or toxoplasmosis as a marker, comorbid or concomitant pathology for babesiosis.

Materials and methods: Blood samples from the patients with revealed borreliosis (Lyme disease) (19 cases), toxoplasmosis (15 cases), rheumatoid arthritis in the stage of exacerbation (10 cases) served as the study material (group 2). In all patients of group 2, positive results for babesiosis were obtained during the polymerase chain reaction. The group of comparative control (group 1) consisted of clinically healthy people $(n=31)$, who underwent the blood cytological examination (light microscopy) preceding the scanning electron microscopy, followed by verification of the results by resources of molecular genetic research (polymerase chain reaction). Scanning electron microscopy was used in this study.

Results: In patients with babesiosis and marker, comorbid and concomitant conditions for this disease (rheumatoid arthritis, borreliosis, toxoplasmosis) it was identified the specific diagnostic criteria for the presence of extraerythrocyte forms of babesia, constant number of erythrocytes and their regenerative forms, the appearance of degenerative forms of erythrocytes with their size and shape pathology. The latter lead to hemodynamic disorders, the development of ischemic and hypoxic changes in tissues of different organs of human body.

Conclusions: Scanning electron microscopy of erythrocytes in patients with babesiosis and marker, comorbid and concomitant conditions for this disease (rheumatoid arthritis, borreliosis, toxoplasmosis) plays the role of an objective method of verifying the results of previous clinical and laboratory diagnosis. The use of scanning electron microscopy allow us to determine in these patients the specific diagnostic criteria for the presence of extraerythrocyte forms of babesia, constant number of erythrocytes and their regenerative forms, degenerative forms of erythrocytes with their size and shape pathology.
\end{abstract}

KEY WORDS: babesiosis, rheumatoid arthritis, borreliosis, toxoplasmosis, erythrocytes, ultramicroscopic characteristics

Wiad Lek. 2021;74(9 p.l):2066-2069

\section{INTRODUCTION}

Babesiosis is an important tick-transmitted disease caused by species of the intra-erythrocytic protozoan Babesia, which is widely distributed across the world [1]. There are more than 100 species of Babesia, all of which have an animal reservoir, typically either rodents or cattle, and are transmitted to humans via a tick vector [2].

Babesiosis imposes a significant health burden, especially on those who are immunocompromised and those who acquire the infection through blood transfusion [3]. In patients with babesiosis may develop various diseases that can be regarded as its markers, act as a comorbid or concomitant pathology. For all babesia infections advanced age and depressed cellular immunity are associated with a higher risk of symptomatic infection and more severe illness [4]. Autoimmune disease like rheumatoid arthritis may develop in patients with babe- siosis and act as a nonspecific marker of this disease [5]. Simultaneous infection with Babesia microti and Borrelia burgdorferi which cases Lyme disease or borreliosis are now the most common tick-transmitted coinfections in the world, and they are a serious health problem because coinfected patients show more intense and persisting disease symptoms [6]. In patients with babesiosis can also develop toxoplasmosis, because infected with Babesia ticks can act as guardians and carriers of Toxoplasma gondii [7].

Our earlier studies [8], studies of other scientists [9] have made it possible to identify the ultramicroscopic features of erythrocytes in persons with babesiosis. However, there are no data in the literature about the erythrocytes characteristics in cases when in patient with babesiosis develops marker, comorbid or concomitant pathology like rheumatoid arthritis, borreliosis or toxoplasmosis. 


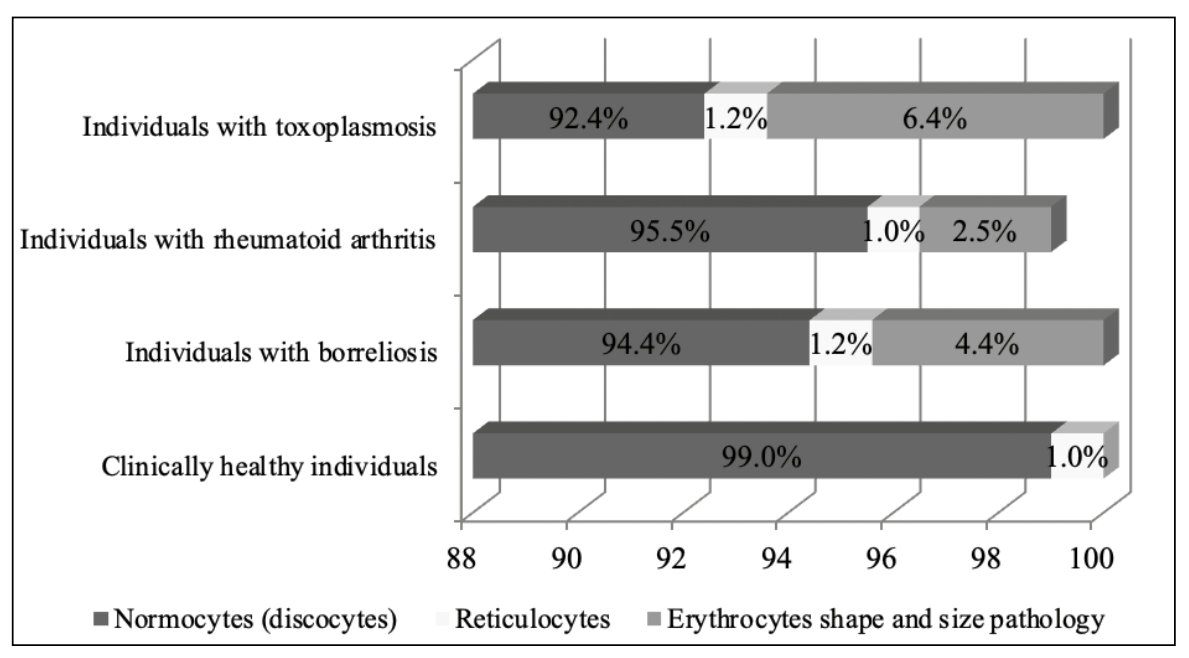

Fig. 1. The relative indicators (\%) of erythrocytes morphological forms in blood in individuals of groups 1 and 2 .

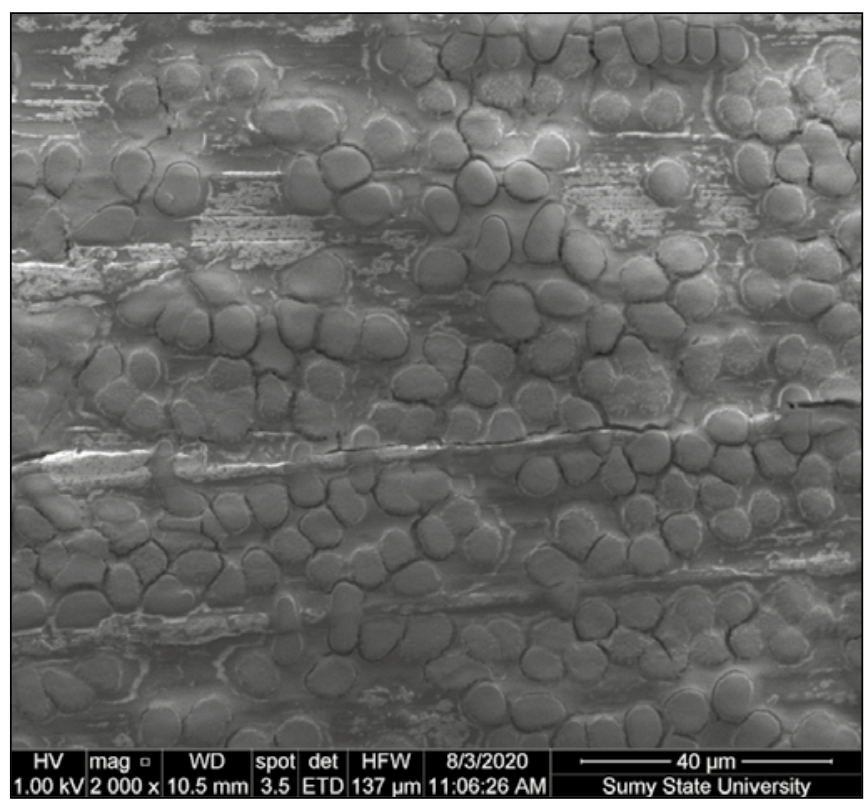

Fig. 2. Erythrocytes of native blood in person with borreliosis. Scanning electron microscopy, $\times 2000$.

\section{THE AIM}

The aim is to determine the ultramicroscopic characteristics of erythrocytes in individuals with rheumatoid arthritis, borreliosis or toxoplasmosis as a marker, comorbid or concomitant pathology for babesiosis.

\section{MATERIALS AND METHODS}

Blood samples from the patients with revealed borreliosis (Lyme disease) (19 cases), toxoplasmosis (15 cases), rheumatoid arthritis in the stage of exacerbation (10 cases) served as the study material (group 2). In all patients of group 2, positive results for babesiosis were obtained during the polymerase chain reaction. The group of comparative control (group 1) consisted of clinically healthy people $(n=31)$, who underwent the blood cytological examination (light microscopy) preceding the scanning electronic microscopy, followed by verification of the results by resources of molecular genetic research (polymerase chain reaction).
The method of scanning electron microscopy was used to study the spatial (three-dimensional coordinate system: the plane formed by the axes OX; OY; OZ) pathology of the size and shape of erythrocytes, microrelief of blood cells surfaces, membranopathies (raptures, macromolecules, conglomerates, micropores). The native peripheral blood smears of various origins were performed in accordance with the generally accepted method without fixation, staining/contrast. For this purpose, $0.01 \mathrm{ml}$ of native blood was applied to the degreased surface of the metal platform $\left(\mathrm{S}=1 \mathrm{~cm}^{2}\right)$, dried in the air of the laboratory premise, analyzed $(\times 2000)$, using the resources of the equipment (SEO-SEM Inspect S50-B; energy dispersive spectrometer AZtecOne with detector X-MaxN20 (Oxford Instruments plc, UC) and power source in the gun: tungsten cathode with thermoelectron emission. This scanning electron microscope made it possible to examine the object under conditions of accelerating voltage of 100 $\mathrm{kV}$. The analysis of the obtained results was performed in comparison.

\section{RESULTS AND DISCUSSION}

Ultramicroscopic analysis of erythrocytes cytoarchitectonics in clinically healthy people demonstrated their mesochromicity, electronic homogeneity of the stroma and presence of a semipermeable integral membrane. The cells were evenly distributed frontally, closely, but not densely, in the form of a rather mesodisperse population. Some loci contained clusters of hypochromic normocytes with enhanced central pallor. The central pallor of the predominant number of normocytes was pronounced, contrasting, occupied less than one third of the diameter and indicated about the native mesochromia, corresponding to the shape of the biconcave disk (normodiskocyte). The tendency to sludge phenomenon (the phenomenon of "coin columns») was minimized. The relative indicators of erythrocytes morphological forms in blood in these individuals specified in figure 1 .

The ultramicroscopic features of erythrocytes revealed by the author in healthy individuals coincide with the data of other scientists [10]. 


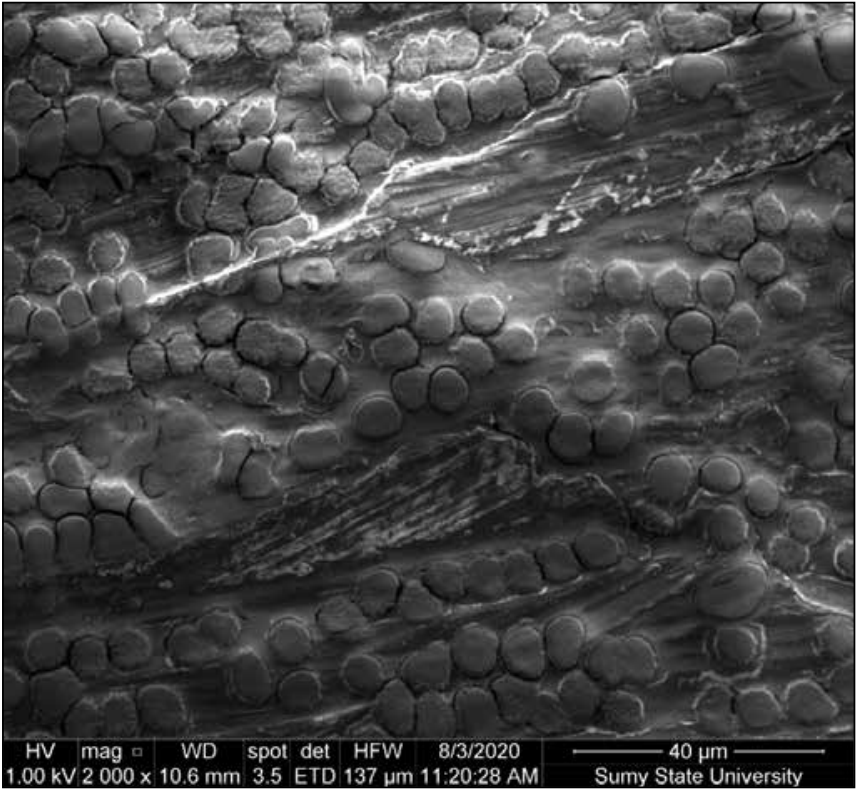

Fig. 3. The erythrocytes in native blood in patient with toxoplasmosis. Scanning electron microscopy, $\times 2000$.

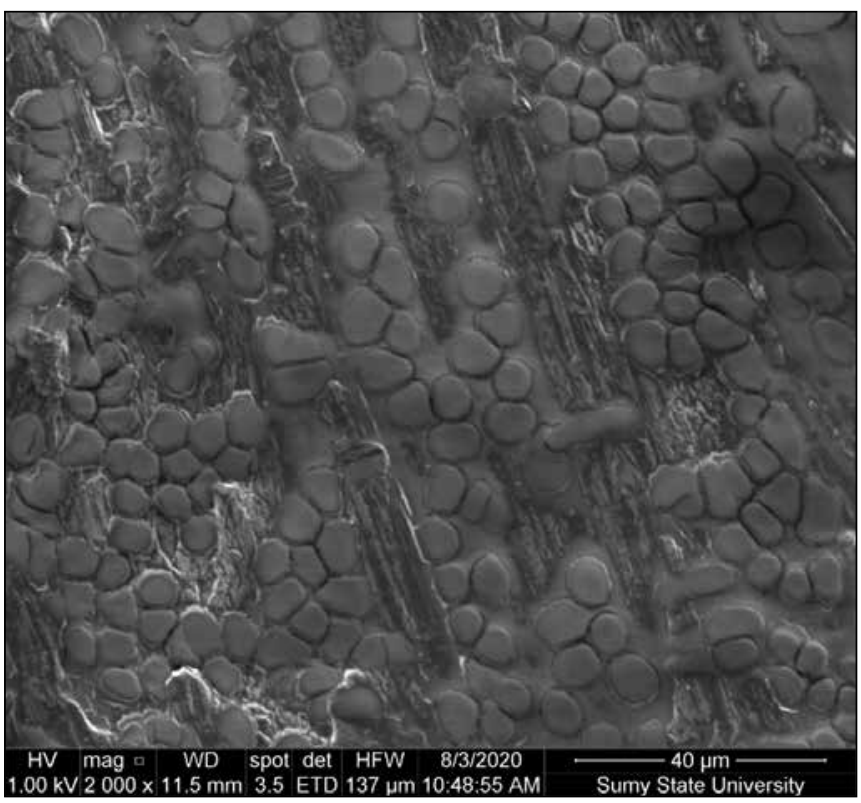

Fig. 4. Erythrocytes in native blood in patient with rheumatoid arthritis. Scanning electron microscopy, $\times 2000$.

In individuals with borreliosis the erythrocyte population was small, located discretely with the cell-free zones present (fig. 2). The density of clusters was insignificant; contacts were preserved in some places. Poikilocytosis was insignificant, confirmed by some forms of schistocytes (triangles, helmets, fragments, pieces). Anisocytosis was highly doubtful. Erythrocytes of irregular shape lined up in chains of up to 11 elements. Erythrophagia was not defined, cell lysis was moderate. Prehemolytic, hemolytic cells have been identified, however, scanty. Cellular detritus was visualized in the form of splintered fragments, different in size and shape surrounded by normocytes. Shadow cells were few. In these individuals, compared with group 1, there were no significant differences in the values of relative indicators of erythrocytes morphological forms in blood (fig. 1).

General ultramicroscopic analysis (scanning electron microscopy, fig. 3) of native blood samples from patients with toxoplasmosis showed that the erythrocyte population of the hematopoietic system was heterogeneous. The dispersion of the cytological spectrum was determined by 5-7 types (normo-, macro-, micro-, ovalo-, schistocytes). Blood corpuscles were located in the frontal plane in clusters, conglomerates and groups (6-15). Single, separately concentrated cells were almost absent. Erythrocyte monolayer was fragmentary with numerous (from 19 to 23 in the field of view of the slide) free from form elements areas of metal platforms. Dense intercellular contacts were absent. "Erythrocyte rosettes", "rosette-like" formations, erythrocyte chains (double) were visualized and expressed. Blood micro- and macrocytes were hypochromic without increase of central pallor, chromocontrast. The formation of sludges (the phenomenon of "coin columns") was observed in some places. Erythrocyte detritus was minimized; the facts of erythrophagia had not been detected. Polychromasia was moderate, constant in nature. The relative indicators of erythrocytes morphological forms in blood did not differ from the indicators of group 1 (fig. 1).

The analysis of the results of scanning ultramicroscopy of native blood slides of patients with rheumatoid arthritis showed that the population of erythrocytes was quite homogeneous (macro--, microcytes), localized frontally as a continuous single layer without conglomerates and clusters (fig. 4). The density of cell contacts was not retained. The boundary between each erythrocyte was expressed, clear, contrasting. The dispersed spectrum of formed blood elements was not wide, their polymorphism was insignificant. The stroma was electronically homogeneous; the membrane was intact, without local and generalized inclusive defects, semipermeable. Central pallor of the vast majority of cells was absent, in the case of differentiation it occupied less than one-third of the diameter. The shadow cells were isolated in the field of view (2-4). Erythrocyte detritus was minimized, apparently, due to timely disposal. However, the facts of erythrophagia were not confirmed. The relative indicators of erythrocytes did not differ from the group 1 indicators (fig. 1).

The determination of pathogens (Babesia spp.) using the routine cytological methods, according to experts, is played the crucial role in confirming the diagnosis of babesiosis [11]. However, the effectiveness of such diagnostic measures is significant in the conditions of acute and subacute disease duration. Instead, cytological diagnosis of asymptomatic forms of infection remains a highly controversial criterion [12].

Scanning electron microscopy is a factor that helps to resolve research controversies in questions about the contribution of parasites in the development of erythrocytes morpho-functional changes [13].

This study allowed us to identify in patients with babesiosis and marker, comorbid and concomitant conditions for this disease 
(rheumatoid arthritis, borreliosis, toxoplasmosis) the specific diagnostic criteria for the presence of extraerythrocyte forms of babesia, constant number of erythrocytes and their regenerative forms, the appearance of degenerative forms of erythrocytes with their size and shape pathology. The latter lead to hemodynamic disorders, the development of ischemic and hypoxic changes in tissues of different organs of human body [14].

\section{CONCLUSIONS}

Scanning electron microscopy of erythrocytes in patients with babesiosis and marker, comorbid and concomitant conditions for this disease (rheumatoid arthritis, borreliosis, toxoplasmosis) plays the role of an objective method of verifying the results of previous clinical and laboratory diagnosis. The use of scanning electron microscopy allow us to determine in these patients the specific diagnostic criteria for the presence of extraerythrocyte forms of babesia, constant number of erythrocytes and their regenerative forms, degenerative forms of erythrocytes with their size and shape pathology.

\section{REFERENCES}

1. Wang F, Jiang JF, Tian J, Du CH. Clinical characteristics, diagnosis and treatment of human babesiosis: a review. Zhongguo Xue Xi Chong Bing Fang Zhi Za Zhi. 2020;33(2):218-224.

2. Saleh AM, Adam SM, Abdel-Motagaly AM, Mohammad A, Ibrahim A, Morsy TA. Human Babesiosis: a general review with special reference to Egypt. Journal of the Egyptian Society of Parasitology. 2015;45(3):493-510.

3. Krause PJ. Human babesiosis. International Journal of Parasitology. 2019;49(2):165-174.

4. Hunfeld KP, Hildebrandt A, Gray JS. Babesiosis: recent insights into an ancient disease. International Journal of Parasitology. 2008;38(11):1219-1237.

5. Horowitz Rl, Freeman PR. Are Mycobacterium drugs effective for treatment resistant Lyme disease, tick-borne co-infections, and autoimmune disease? JSM Arthritis. 2016; 1(2): 1008.

6. Djokic V, Primus S, Akoolo L, Chakraborti M, Parveen N. Age-related differential stimulation of immune response by Babesia microti and Borrelia burgdorferi during acute phase of infection affects disease severity. Frontiers in Immunology. 2018;9:2891. doi: 10.3389/ fimmu.2018.02891.

7. Asman M, Solarz K, Cuber P, Gąsior T, Szilman P, Szilman E, Tondaś E, Matzullok A, Kusion N, Florek K. Detection of protozoans Babesia microti and Toxoplasma gondii and their co-existence in ticks (Acari: Ixodida) collected in Tarnogórski district (Upper Silesia, Poland). Annals of Agricultural and Environmental Medicine. 2015;22(1):80-83.

8. Torianyk II. Ultramicroscopis erythrocytes profile as a component of the babesiosis pathogenesis. Wiad. Lek. 2021;74(4):911-914.

9. Akel T, Mobarakai N. Hematologic manifestations of babesiosis. Annals of Clinical Microbiology and Antimicrobials. 2017;16(1):6. doi: 10.1186/ s12941-017-0179-z.

10. Ghosh S, Chakraborty I, Chakraborty M, Mukhopadhyay A, Mishra $R$, Sarkar D. Evaluating the morphology of erythrocyte population: an approach based on atomic force microscopy and flow cytometry. Biochimica et Biophysica Acta. 2016;1858(4):671-681.
11. Huber D, Beck A, AnzulovićŽ, JurkovićD, Polkinghorne A, Baneth G, Beck R. Microscopic and molecular analysis of Babesia canis in archived and diagnostic specimens reveal the impact of anti-parasitic treatment and postmortem changes on pathogen detection. Parasit Vectors. 2017 0ct 18;10(1):495. doi: 10.1186/s13071-017-2412-1.

12. Krause PJ, Auwaerter PG, Bannuru RR, Branda JA, Falck-Ytter YT, Lantos PM, Lavergne V, Meissner HC, Osani MC, Rips JG, Sood SK, Vannier E, Vaysbrot EE, Wormser GP. Clinical practice guidelines by the Infectious Diseases Society of America (IDSA): 2020 Guideline on diagnosis and management of Babesiosis. Clinical Infectious Diseases. 2021;72(2):e49-e64. doi: 10.1093/cid/ciaa1216.

13. Xia Z, Hui W, Jing-Bo X, Shang X, Xiao-Nong Z. Epidemic and research progress of babesiosis. Zhongguo Xue Xi Chong Bing Fang Zhi Za Zhi. 2019;31(1):63-70.

14. Fedulenkova YY, Myroshnychenko MS, Astapieva OM, Fatieiev AA, Akritova KO, Molodan DV. Characteristic of autopsy material in cases of chronic diffuse pathology of the liver parenchyma diagnosed during patient's lifetime using the ultrasound method. Wiad. Lek. 2020;73(9 (z. 2):1995-1999.

\section{Acknowledgments:}

The author of the study expresses her sincere gratitude to the director of the Center of Collective Use of Scientific Equipment "Laboratory of Materials Science of Helioenergy, Sensory and Nanoelectronic Systems» of Sumy State University V. Zhurba; scientists of the State Institution "Mechnikov Institute of Microbiology and Immunology of the National Academy of Medical Sciences of Ukraine» (O. Gryshyna, T. Davydova, T.Osolodchenko); orthopedist-traumatologist of the Public Nonprofit Organization of the Kharkiv District Council «Regional Clinical Hospital» (O. Kaminsky); villagers of the summer cottages of Merlo, Kuryly, Skovorodynivka (Bogodukhiv district, Kharkiv region, Ukraine) for the technical part of the research and valuable reasonable recommendations.

ORCID and contributionship:

Inna I. Torianyk: 0000-0001-6843-8808 A-F

\section{Conflict of interest:}

The Author declare no conflict of interest.

\section{CORRESPONDING AUTHOR Inna I. Torianyk}

Laboratory of Viral Infections,

State Institution «Mechnikov Institute of Microbiology and Immunology of the National Academy of Medical Sciences of Ukraine» 39 Kamysheva Ivana str., apt. 9, 61038, Kharkiv, Ukraine e-mail: patholognew@ukr.net

Received: 29.06 .2021

Accepted: 25.08 .2021

A - Work concept and design, B - Data collection and analysis, C - Responsibility for statistical analysis,

D-Writing the article, $\mathbf{E}$-Critical review, $\mathbf{F}$ - Final approval of the article 